\title{
Bioinformatics on $\beta$-Barrel Membrane Proteins: Sequence and Structural Analysis, Discrimination and Prediction
}

\author{
M. Michael Gromiha \\ Computational Biology Research Center (CBRC) \\ National Institute of Advanced Industrial Science and Technology (AIST) \\ AIST Tokyo Waterfront Bio-IT Research Building \\ 2-42 Aomi, Koto-ku, Tokyo 135-0064, Japan \\ michael-gromiha@aist.go.jp
}

\begin{abstract}
The analysis on the amino acid sequences of transmembrane beta barrel proteins (TMBs) provides deep insights about their structure and function. We found that the occurrence of Ser, Asn and Gln is significantly higher in TMBs than globular proteins, which might be due to their importance in the formation of $\beta$-barrel structures in the membrane, stability of binding pockets and the function of TMBs. Utilizing this information, we have devised statistical methods and machine learning techniques to discriminate TMBs from other folding types of globular and membrane proteins and we obtained the maximum accuracy of $96 \%$. Further, we have devised protocols for identifying the membrane spanning $\beta$-strand segments and detecting TMBs in genomic sequences.
\end{abstract}

Keywords: $\beta$-barrel membrane protein, amino acid composition, sequence analysis, discrimination, prediction, genome.

\section{Introduction}

The $\beta$-barrel membrane proteins (TMBs) perform a variety of functions, such as mediating non-specific, passive transport of ions and small molecules, selectively passing the molecules like maltose and sucrose and are involved in voltage dependent anion channels. These proteins contain $\beta$-strands as their membrane spanning segments and are found in the outer membranes of bacteria, mitochondria and chloroplast. The assembly of TMBs is somewhat more complex when compared to the assembly of transmembrane helical proteins having $\alpha$-helices as transmembrane parts. This is probably due to the difference of amino acid sequences in the transmembrane part strands and helices; transmembrane helical proteins $(\mathrm{TMH})$ contain a stretch of hydrophobic amino acid residues whereas transmembrane strand proteins are intervened by several charged and polar residues. Because of this feature, most predictive schemes, which are successful in predicting transmembrane helical segments, fail to predict the transmembrane strand segments and discriminating $\beta$ barrel membrane proteins. 
We have systematically analyzed the amino acid compositions of TMBs, TMH and globular proteins, and observed that the residues Ser, Asn and Gln are predominant in TMBs. Utilizing amino acid and dipeptide compositions, we have devised statistical methods and machine learning techniques for discriminating TMBs. Further, we have developed a rule based approach and neural networks based method for identifying the membrane spanning segments. A novel protocol has been proposed for detecting TMBs in genomic sequences and a database has been set up for the annotation of TMBs in genomes.

\section{Materials and Methods}

\subsection{Dataset}

We have constructed several sets of data for the analysis, discrimination and prediction: (i) a dataset of 377 well annotated TMB sequences obtained from PSORT database (1) and a subset of 208 non-redundant TMB sequences with less than $40 \%$ sequence identity obtained with CD-HIT algorithm (2), (ii) non-redundant dataset of 19 known TMB structures with the sequence identity of less than $25 \%$, (iii) 674 globular proteins belonging to different structural classes (155 all- $\alpha, 156$ all- $\beta, 184$ $\alpha+\beta$ and $179 \alpha / \beta$ proteins), (iv) non-redundant data set of 1602 globular proteins belonging to 30 different folds obtained from Protein Data Bank (3) (v) a dataset of 268 well-annotated TMH sequences and a subset of 206 non-redundant TMH sequences obtained from PSORT and (vi) the amino acid sequences of 275 completed genomes from NCBI database (http://www.ncbi.nih.gov/). This includes 23 genomes from archaea, 237 from bacteria and 15 from eukaryote. The total number of proteins in these three kingdoms of life is 52241, 686562 and 165186, respectively with the total of 903,989 sequences.

\subsection{Computation of Amino Acid and Dipeptide Compositions}

The amino acid composition for a residue type (e.g. Ala) in a protein is the number of amino acids of specific type normalized with the total number of residues. It is defined as:

$$
\operatorname{Comp}(\mathrm{i})=\Sigma \mathrm{n}_{\mathrm{i}} / \mathrm{N}
$$

where, $\mathrm{i}$ stands for the 20 amino acid residues. $n_{i}$ is the number of residues of each type and $\mathrm{N}$ is the total number of residues.

The composition of dipeptides is a measure to quantify the preference of amino acid residue pairs in a sequence. This has been computed using the following expression:

$$
\operatorname{Dipep}(\mathrm{i}, \mathrm{j})=\Sigma \mathrm{N}_{\mathrm{ij}} * 100 /\left(\Sigma \mathrm{N}_{\mathrm{i}}+\Sigma \mathrm{N}_{\mathrm{j}}\right)
$$

where $\mathrm{i}, \mathrm{j}$ stands for the distribution of 20 amino acid residues at positions $\mathrm{i}$ and $\mathrm{i}+1$. $\mathrm{N}_{\mathrm{i}, \mathrm{j}}$ is the number of residues of type $\mathrm{i}$ followed by the residue j. $\Sigma \mathrm{N}_{\mathrm{i}}$ and $\Sigma \mathrm{N}_{\mathrm{j}}$ are the total number of residues of type $i$ and $j$, respectively. 
The concept of motifs provides the information about the preference of residue pairs with a gap (any residue between the pair of residues). This has been computed using the same expression that we used for dipeptide composition (Eqn. 2). The main difference is that the residues $\mathrm{i}$ and $\mathrm{j}$ are the distribution of 20 amino acid residues at positions $i$ and $i+1$ for dipeptides, and $i$ and $i+2$, $i$ and $i+3$ etc. for motifs.

\subsection{Discrimination Methods}

We have used statistical methods and machine learning algorithms for discriminating TMBs. In these methods, we have used the compositions of amino acids and dipeptides as attributes. The protocol used to discriminate TMBs using amino acid composition is given below: The amino acid composition has been computed for standard datasets of both globular $\left(\mathrm{Comp}_{\mathrm{glob}}\right)$ and TMBs $\left(\mathrm{Comp}_{\mathrm{TMB}}\right)$. For a new protein, $X$, firstly, we have calculated the amino acid composition using Eqn. 1. Then we have calculated the total absolute difference of amino acid composition between protein $\mathrm{X}$ and the amino acid composition of globular proteins, and that between protein $\mathrm{X}$ and TMBs. The protein $\mathrm{X}$ is predicted to be a TMB if the deviation is lowest with Comp $\mathrm{TMB}_{\mathrm{TMB}}$ and vice versa (4).

We have followed the below mentioned steps to discriminate TMBs using residue pair preference/motif: (i) calculated the dipeptide composition for both globular (Dipep $\left._{\text {glob }}\right)$ and TMBs (Dipep $\left.{ }_{\mathrm{TMB}}\right)$ and the difference between them $\left(\sigma_{\mathrm{TMB} \text {-glob }}\right)$; (ii) for a new protein, $X$, they have calculated the dipeptide composition using Eqn. 2 and given weights to the dipeptide composition with $\sigma_{\mathrm{TMB} \text {-glob }}$; (iii) calculated the sum of weighted dipeptide composition and (iv) the protein $\mathrm{X}$ is predicted to be an TMB if the total weighted dipeptide composition is positive and globular protein otherwise.

Further, machine learning techniques including Bayes functions, Neural networks, Logistic functions, Support vector machines, Regression analysis, Nearest neighbor methods, Meta learning, Decision trees and Rules have been used to discriminate the TMBs.

\subsection{Assessment of the Validity of the Method}

We have performed a 5-fold cross-validation test for assessing the validity of the present work. In this method, the data set is divided into five groups, four of them are used for training and the rest is used for testing the method. The same procedure is repeated for five times and the average is computed for obtaining the accuracy of the method.

We have used different measures to assess the accuracy of discriminating TMBs, non-TMBs and combination of the two. The term, sensitivity shows the correct prediction of TMBs, specificity about the non-TMBs and accuracy indicates the overall assessment. These terms are defined as follows:

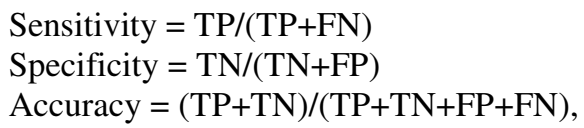

where, TP, FP, TN and FN refer to the number of true positives (TMBs identified as TMBs), false positives (non-TMBs identified as TMBs), true negatives (non-TMBs 
identified as non-TMBs), and false negatives (TMBs identified as non-TMBs), respectively.

\section{Results and Discussion}

\subsection{Amino Acid Composition}

We have computed the amino acid compositions for the 20 amino acid residues in 377 TMBs and the results are displayed in Fig. 1. In this figure, we have also included the data for 674 globular proteins for comparison. We observed that the composition of Glu, His, Ile and Cys are higher in globular proteins than TMBs and an opposite trend is observed for Ser, Asn and Gln (4). The formation of disulfide bonds between Cys residues requires an oxidative environment and such disulfide bridges are not usually found in intracellular proteins (5). Further, the analysis on the three-dimensional structures of $15 \beta$-barrel TMBs showed the presence of just eight $(0.1 \%)$ Cys residues and none of them are in membrane part. Hence, the occurrence of Cys is significantly higher in globular proteins than in TMBs. Glu is a strong helix former and this tendency influences the higher occurrence of it in globular proteins than TMBs. The comparative analysis on the occurrence of Ile in the $\beta$-strand segments of globular and TMBs revealed that the preference of Ile in TMBs is less than that in globular proteins, which may increase the occurrence of it in globular proteins.

The structural analysis of several TMBs shows that the residues, Ser, Asn and Gln play an important role to the stability and function of TMBs. In OmpA, the interior of $\beta$-strands contain an extended hydrogen bonding network of charged and polar residues and especially, the side chains of the residues, Ser22, Gln228 and Asn258 in OmpT, located above the membrane form hydrogen bonds to main chain atoms in the $\beta$-barrel. Interestingly, none of the residues, which have high composition in globular proteins (Glu, His, Ile and Cys), are involved in such pattern (6,7). In FecA, Yue et al. (8) showed that the binding pockets for diferric dicitrate involve the hydrogen bonds from the three residues, Gln176, Gln570 and Asn721 as shown in Figure 2. Similar

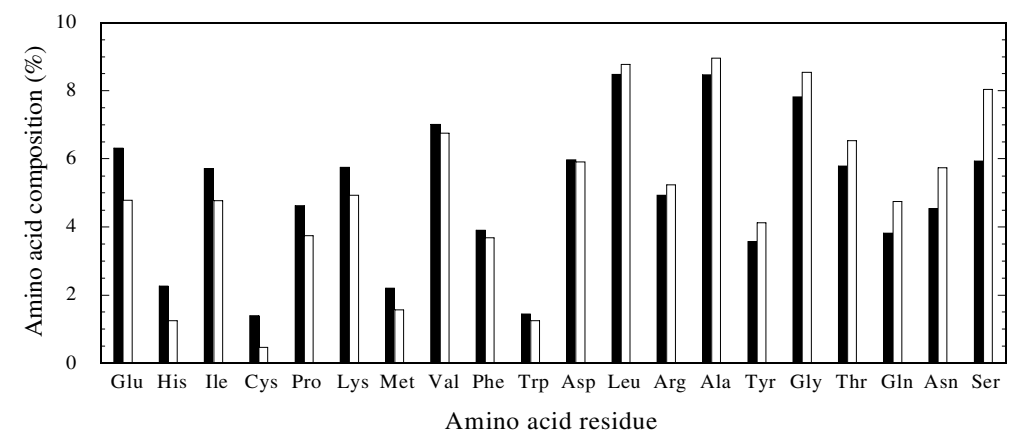

Fig. 1. Amino acid composition of the 20 amino acid residues in globular (filled bars) and TMBs (open bars) 


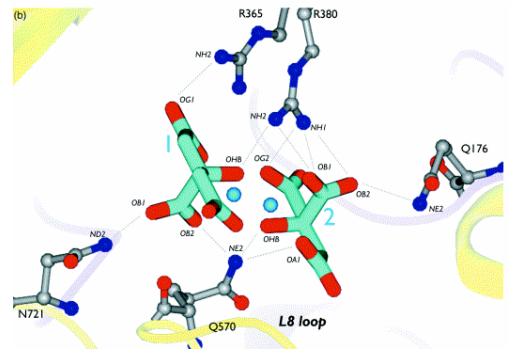

Fig. 2. The binding pockets for diferric dicitrate in FecA. The hydrogen bonds are shown as dotted lines. trend is observed in other TMBs and this analysis revealed that the high occurrence of Ser, Asn and Gln in TMBs is required in the formation of $\beta$-barrel structures in the membrane, stability of binding pockets and the function of TMBs.

\subsection{Discrimination of $\beta$-Barrel Membrane Proteins}

We have used the compositions of amino acids, dipeptides and motifs for discriminating TMBs using statistical methods. An example to discriminate

TMBs using amino acid composition is shown in Table 1.

For 1ADT (adenovirus DNA binding protein), the deviation of amino acid composition from globular protein (34.18) is less than that of TMB (39.89) and hence this protein is predicted as a non-TMB. On the other hand, for OutD protein, the deviation from TMB (16.09) is less than that from globular protein (23.70) and hence it is identified as an TMB.The amino acid composition based method could correctly identify $89 \%$ of the TMBs (334/377) and exclude $79 \%$ of globular proteins $(531 / 674)$.

Table 1. Steps to discriminate globular and outer membrane proteins in two typical proteins

\begin{tabular}{|c|c|c|c|c|c|c|c|c|}
\hline Residue & $\mathrm{N}$ & Comp & $\sigma_{\text {glob }}$ & $\sigma_{\mathrm{TMB}}$ & $\mathrm{N}$ & Comp & $\sigma_{\text {glob }}$ & $\sigma_{\mathrm{TMB}}$ \\
\hline \multicolumn{5}{|c|}{ Adenovirus DNA-Binding Protein (1ADT) } & \multicolumn{3}{|c|}{ OutD protein } & \\
\hline Ala & 10 & 11.11 & 2.64 & 2.16 & 54 & 8.31 & 0.16 & 0.64 \\
\hline Asp & 4 & 4.44 & 1.53 & 1.47 & 40 & 6.15 & 0.18 & 0.24 \\
\hline Cys & 1 & 1.11 & 0.28 & 0.64 & 1 & 0.15 & 1.24 & 0.32 \\
\hline Glu & 7 & 7.78 & 1.46 & 3.00 & 31 & 4.77 & 1.55 & 0.01 \\
\hline Phe & 5 & 5.56 & 1.65 & 1.88 & 21 & 3.23 & 0.68 & 0.45 \\
\hline Gly & 3 & 3.33 & 4.49 & 5.21 & 46 & 7.08 & 0.74 & 1.46 \\
\hline His & 4 & 4.44 & 2.18 & 3.19 & 3 & 0.46 & 1.80 & 0.79 \\
\hline Ile & 1 & 1.11 & 4.60 & 3.66 & 35 & 5.38 & 0.33 & 0.61 \\
\hline Lys & 7 & 7.78 & 2.02 & 2.85 & 28 & 4.31 & 1.45 & 0.62 \\
\hline Leu & 10 & 11.11 & 2.63 & 2.33 & 53 & 8.15 & 0.33 & 0.63 \\
\hline Met & 4 & 4.44 & 2.23 & 2.88 & 19 & 2.92 & 0.71 & 1.36 \\
\hline Asn & 4 & 4.44 & 0.10 & 1.30 & 43 & 6.62 & 2.08 & 0.88 \\
\hline Pro & 3 & 3.33 & 1.30 & 0.41 & 21 & 3.23 & 1.40 & 0.51 \\
\hline Gln & 4 & 4.44 & 0.62 & 0.31 & 33 & 5.08 & 1.26 & 0.33 \\
\hline Arg & 3 & 3.33 & 1.60 & 1.91 & 37 & 5.69 & 0.76 & 0.45 \\
\hline Ser & 3 & 3.33 & 2.61 & 4.72 & 55 & 8.46 & 2.52 & 0.41 \\
\hline Thr & 6 & 6.67 & 0.88 & 0.13 & 47 & 7.23 & 1.44 & 0.69 \\
\hline Val & 6 & 6.67 & 0.35 & 0.09 & 64 & 9.85 & 2.83 & 3.09 \\
\hline Trp & 2 & 2.22 & 0.78 & 0.98 & 6 & 0.92 & 0.52 & 0.32 \\
\hline Tyr & 3 & 3.33 & 0.25 & 0.80 & 12 & 1.85 & 1.73 & 2.28 \\
\hline Total & & & 34.18 & 39.89 & & & 23.70 & 16.09 \\
\hline Discrimi & \multicolumn{4}{|c|}{ Globular protein } & \multicolumn{4}{|c|}{$\beta$-barrel membrane protein } \\
\hline
\end{tabular}

$\mathrm{N}$ : number of residues; $\sigma_{\mathrm{glob}}=|\operatorname{comp}-\operatorname{comp}(\mathrm{glob})| ; \sigma_{\mathrm{TMB}}=|\operatorname{comp}-\operatorname{comp}(\mathrm{TMB})|$. 
The dipeptides have more information than just amino acid composition and we observed an increase in accuracy. This information could correctly identify $95 \%$ of the TMBs and exclude $79 \%$ of the globular proteins (9). The performance of motifs is better to exclude globular proteins and the accuracies of identifying 377 TMBs and excluding 674 globular proteins are $95.8 \%$ and $82.2 \%$, respectively (10).

Further, we have analyzed different machine learning techniques for discriminating TMBs. These methods could discriminate a set of 1088 TMBs and globular proteins with the accuracy in the range of $89-92 \%$ using amino acid composition (11). We have also used a set of 49 amino acid properties for discrimination, which improved the accuracy up to $94 \%$ for the same set of proteins (12). Interestingly, this will also have the ability of correctly excluding 1612 proteins belonging to 30 major folds of globular proteins with the accuracy of $99 \%$ as seen in Figure 3. The inclusion of PSSM profiles enhanced the accuracy of discriminating TMBs (in a dataset of 206 TMBs and 1045 non-TMBs obtained with less than $40 \%$ sequence identity) up to $96.4 \%$ (13).

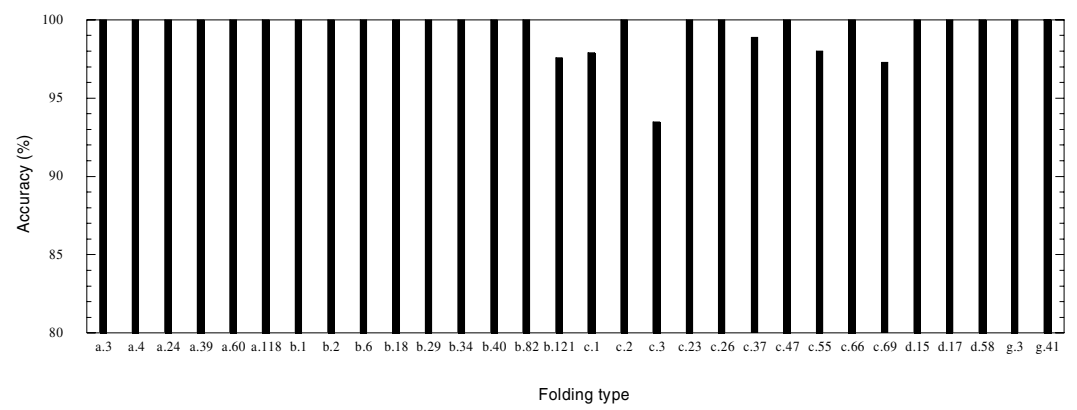

Fig. 3. The accuracy of excluding 30 major folding types of globular proteins. The SCOP classification (14) is used to denote the folding types.

Table 2. Predictive performance of different methods for discriminating TMBs

Amino acid composition ( $\beta$-strand segments)

Amino acid composition (TMB and globular proteins) 87

Hidden Markov model and alignment profies

Hidden Markov model

Neural networks (amino acid composition)

Support vector machines (amino acid composition)

Support vector machines (amino acid and dipeptide compositions) 94 
The comparison of statistical and machine learning techniques for discriminating TMBs is presented in Table 2. We noticed that the accuracy is remarkably higher with machine learning techniques than with statistical methods. Further, the statistical methods could correctly identify the TMBs whereas the performance of machine learning techniques is better for excluding globular and TMH proteins than identifying TMBs.

\subsection{Prediction of Membrane Spanning Segments}

We have developed a "rule based approach" for predicting transmembrane $\beta$-strands using three features, (i) preference of amino acid residues in membrane spanning $\beta$ strands (conformational parameters), (ii) hydrophobic character and (iii) amphipathicity (21). A set of five primary rules have been designed to assign the priority of each residue to be in transmembrane $\beta$-strand and four secondary rules to pick up the membrane spanning segments. The primary rules for assigning the priority of each residue, $i$, are: $\beta(\mathrm{i})>1.0$ (average conformational parameter), $1 / 6 \sum_{i=1}^{6} \beta(i)>$ 1.0, $\mathrm{H}_{\mathrm{p}}(\mathrm{i})>13.34$ (average hydrophobicity), $1 / 6 \sum_{i=1}^{6} H_{p}(i)>13.34$ and $1 / 2 \sum_{i=1}^{2} H_{p}(i)$ $=13.34 \pm 0.5$ (oscillating around the average hydrophobicity). If these conditions are satisfied the priority is one and zero, otherwise. The secondary rules for picking up the membrane spanning segments are: if any residue has the priority of 5, two consecutive residues have the priority of 4 or three consecutive residues have the priority of $\geq 3$ there is a possibility of a transmembrane $\beta$-strand segment around the residue(s). Extend the length in both directions so that there may not be two consecutive low priority residues (less than 3 ) or a residue of zero priority. If the segment is longer than 20 residues cut into two smaller segments at the residue of highest hydrophobicity. This method is mainly applicable to bacterial porins and it could predict the membrane spanning segments with the accuracy of $82 \%$.

Further, we have set up a method using neural networks for predicting membrane spanning regions in TMBs. In this method, a three-layered neural network with one hidden layer has been used for predictions. Input layer reads the input information about a residue and its sequence neighbors from the neural network through a running window. Each residue is represented by a 21-bit vector ( 20 units for the amino acids and one unit for describing the terminal position of the protein). This input information is then fed forward through linear activation function, and the final signal received at the single unit of the output layer is transformed via a sigmoidal function to yield a value between 0 and 1 . Our method could predict the membrane spanning regions of $13 \mathrm{TMBs}$ with the accuracy of $73 \%$ using only the sequence information (22). In addition, our method would provide the probability of each residue to be in the transmembrane segment.

\subsection{Annotation of $\beta$-Barrel Membrane Proteins in Genomic Sequences}

We have developed a novel method for detecting TMBs in genomic sequences. We have followed the below mentioned steps for detecting TMBs as depicted in Figure 4: (i) identification of TMBs using the preference of residue pairs in globular, TMH and 
TMBs, (ii) exclusion of TMH proteins using SOSUI, a prediction system for $\mathrm{TMH}$ proteins, (iii) elimination of globular/TMH proteins that show the sequence identity of more than $70 \%$ for the coverage of $80 \%$ residues with known structures in PDB and (iv) elimination of globular/TMH proteins that have the sequence identity of more than $60 \%$ with known sequences in SWISS-PROT. This method showed good agreement with experimental observations. An example is shown below for E. coli.

The complete genome of E. coli has 4237 proteins and the comparison of residue pair preferences identified 1036 proteins as TMBs (step i). Further, globular and TMH proteins were eliminated with steps (ii-iv) and finally we obtained 87 sequences as TMBs. Interestingly, all the 11 TMBs of known structures from $\mathrm{E}$. coli have been identified by our method. Further, our approach could detect representative sequences in all the 15 families of TMBs deposited in Transport Classification Database (23).

We have developed a database, TMBETA-GENOME, for annotated TMBs in 275 genomic sequences and it is available at http://tmbeta-genome.cbrc.jp/ annotation/. TMBETA-GENOME includes several features, such as, the service for detecting TMBs in genomic sequences using various methods, related references, statistics for the detected TMBs by different methods for each genome, details about all algorithms used to detect TMBs, relative links to other databases and a help page (24). An example is shown in Figure 5. In this figure the results are shown for Escherichia coli. K12 genome. The method, "New approach" has been selected for obtaining the annotated TMBs. This search picked up 87 entries and the TMBs identified by the new approach are shown with the identification number. In addition, the results obtained with other methods are also given for comparison. This database is a valuable resource for finding annotated TMBs in genomic sequences.

\section{TMB finding pipeline}

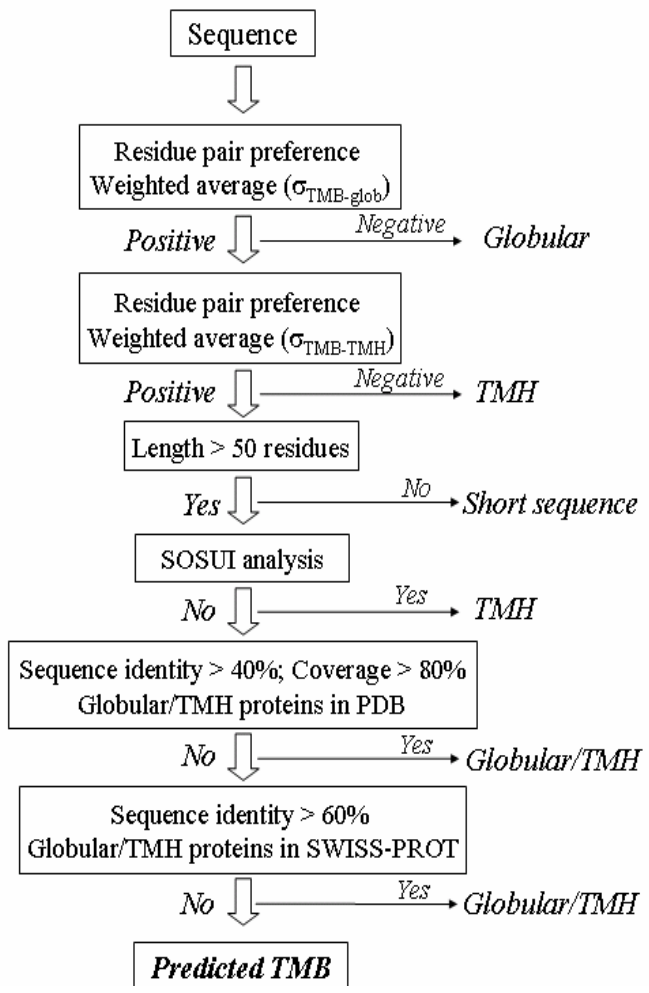

Fig. 4. Pipeline for detecting TMB proteins in genomic sequences 


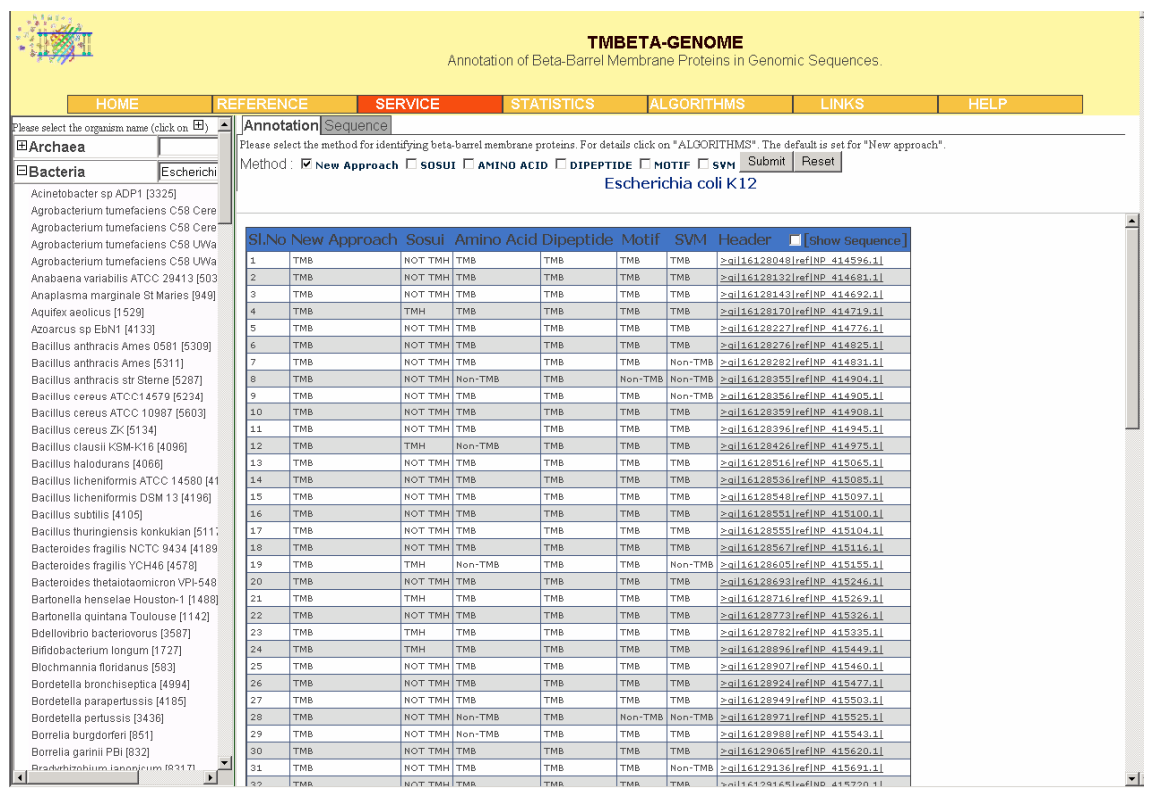

Fig. 5. Annotated TMBs in E. coli using our approach and deposited in TMBETA-GENOME database

\section{Conclusions}

We have systematically analyzed the characteristic features of amino acid residues in the sequences of TMBs and globular proteins and revealed the differences between them. Utilizing this information, we have developed statistical and machine learning techniques for discriminating TMBs from other folding types of globular and membrane proteins. Further, rule based and neural networks methods have been proposed for identifying membrane spanning segments. A new approach has been developed to detect TMBs in genomic sequences and a data base has been set up for the annotated TMBs in genomes.

Acknowledgements. The author wishes to thank Dr. Makiko Suwa, Dr. Shandar Ahmad and Dr. Yukimitsu Yabuki for helpful discussions. The travel support received from Japan Society for the Promotion of Sciences (JSPS) is gratefully acknowledged.

\section{References}

1. Gardy, J.L., Spencer, C., Wang, K., Ester, M., Tusnady, G.E., Simon, I., Hua, S., de Fays, K., Lambert, C., Nakai, K., Brinkman, F.S.: PSORT-B: Improving protein subcellular localization prediction for Gram-negative bacteria. Nucleic Acids Res. 31, 3613-3617 (2003)

2. Li, W., Jaroszewski, L., Godzik, A.: Clustering of highly homologous sequences to reduce the size of large protein databases. Bioinformatics 17, 282-283 (2001)

3. Berman, H.M., Westbrook, J., Feng, Z., Gilliland, G., Bhat, T.N., Weissig, H., Shindyalov, I.N., Bourne, P.E.: The Protein Data Bank. Nucleic Acids Res. 28, 235-242 (2000) 
4. Branden, C., Tooze, C.: Introduction to protein structure. Garland Publishing Inc., New York (1999)

5. Gromiha, M.M., Suwa, M.A.: Simple statistical method for discriminating outer membrane proteins with better accuracy. Bioinformatics 21, 961-968 (2005)

6. Pautsch, A., Schulz, G.E.: High-resolution structure of the OmpA membrane domain. J. Mol. Biol. 298, 273-282 (2000)

7. Vandeputte-Rutten, L., Kramer, R.A., Kroon, J., Dekker, N., Egmond, M.R., Gros, P.: Crystal structure of the outer membrane protease OmpT from Escherichia coli suggests a novel catalytic site. EMBO J. 20, 5033-5039 (2001)

8. Yue, W.W., Grizot, S., Buchanan, S.K.: Structural evidence for iron-free citrate and ferric citrate binding to the TonB-dependent outer membrane transporter FecA. J. Mol. Biol. 332, 353-368 (2003)

9. Gromiha, M.M., Ahmad, S., Suwa, M.: Application of residue distribution along the sequence for discriminating outer membrane proteins. Comput. Biol. Chem. 29, 135-142 (2005)

10. Gromiha, M.M.: Motifs in outer membrane protein sequences: Applications for discrimination. Biophys. Chem. 117, 65-71 (2005)

11. Gromiha, M.M., Suwa, M.: Discrimination of outer membrane proteins using machine learning algorithms. Proteins: Struct. Funct. Bioinf. 63, 1031-1037 (2006)

12. Gromiha, M.M., Suwa, M.: Influence of amino acid properties for discriminating outer membrane proteins at better accuracy. Biochim. Biophys. Acta 1764, 1493-1497 (2006)

13. Ou, Y.-Y., Gromiha, M.M., Chen, S.-A., Suwa, M.: Discrimination of beta barrel membrane proteins using RBF networks and PSSM profiles. Proteins: Struct. Funct. Bioinf. (in press)

14. Murzin, A.G., Brenner, S.E., Hubbard, T., Chothia, C.: SCOP: a structural classification of proteins database for the investigation of sequences and structures. J. Mol. Biol. 247, 536540 (1995)

15. Gnanasekaran, T.V., Peri, S., Arockiasamy, A., Krishnaswamy, S.: Profiles from structure based sequence alignment of porins can identify beta stranded integral membrane proteins. Bioinformatics 16, 839-842 (2000)

16. Liu, Q., Zhu, Y., Wang, B., Li, Y.: Identification of beta-barrel membrane proteins based on amino acid composition properties and predicted secondary structure. Comput. Biol. Chem. 27, 355-361 (2003)

17. Martelli, P.L., Fariselli, P., Krogh, A., Casadio, R.: A sequence-profile-based HMM for predicting and discriminating beta barrel membrane proteins. Bioinformatics 18, S46-S53 (2002)

18. Bagos, P.G., Liakopoulos, T.D., Spyropoulos, I.C., Hamodrakas, S.J.: A Hidden Markov Model method, capable of predicting and discriminating beta-barrel outer membrane proteins. BMC Bioinformatics 5, 29 (2004)

19. Park, K.J., Gromiha, M.M., Horton, P., Suwa, M.: Discrimination of outer membrane proteins using support vector machines. Bioinformatics 21, 4223-4229 (2005)

20. Garrow, A.G., Agnew, A., Westhead, D.R.: TMB-Hunt: a web server to screen sequence sets for transmembrane beta-barrel proteins. Nucleic Acids Res. 33, W188-W192 (2005)

21. Gromiha, M.M., Majumdar, R., Ponnuswamy, P.K.: Identification of membrane spanning beta strands in bacterial porins. Protein Eng. 10, 497-500 (1997)

22. Gromiha, M.M., Ahmad, S., Suwa, M.: TMBETA-NET: Discrimination and prediction of membrane spanning?-strands in outer membrane proteins. Nucleic Acids Res. 33, W164W167 (2005)

23. Busch, W., Saier Jr., M.H.: The transporter classification (TC) system, 2002. Crit. Rev. Biochem. Mol. Biol. 37, 287-337 (2002)

24. Gromiha, M.M., Yabuki, Y., Kundu, S., Suharnan, S., Suwa, M.: TMBETA-GENOME: database for annotated beta-barrel membrane proteins in genomic sequences. Nucleic Acids Res. 35, D314-D316 (2007) 